\title{
On the dissolution of cellulose in tetrabutylammonium acetate/dimethyl sulfoxide: a frustrated solvent
}

\author{
Alexander Idström • Luigi Gentile • Marta Gubitosi • Carina Olsson • \\ Björn Stenqvist • Mikael Lund • Karl-Erik Bergquist • Ulf Olsson • \\ Tobias Köhnke $\cdot$ Erik Bialik (i)
}

Received: 4 March 2017/Accepted: 22 May 2017/Published online: 21 June 2017

(C) The Author(s) 2017. This article is an open access publication

\begin{abstract}
We have found that the dissolution of cellulose in the binary mixed solvent tetrabutylammonium acetate/dimethyl sulfoxide follows a previously overlooked near-stoichiometric relationship such that one dissolved acetate ion is able to dissolve an amount of cellulose corresponding to about one glucose residue. The structure and dynamics of the resulting cellulose solutions were investigated using small-angle X-ray scattering (SAXS) and nuclear magnetic resonance techniques as well as molecular dynamics simulation. This yielded a detailed picture
\end{abstract}

Electronic supplementary material The online version of this article (doi:10.1007/s10570-017-1370-2) contains supplementary material, which is available to authorized users.

B. Stenqvist · M. Lund · E. Bialik ( $₫)$

Division of Theoretical Chemistry, Department of

Chemistry, Lund University, P.O. Box 124, 22100 Lund,

Sweden

e-mail: erik.bialik@mail.com

L. Gentile · M. Gubitosi · U. Olsson

Division of Physical Chemistry, Department of

Chemistry, Lund University, P.O. Box 124, 22100 Lund,

Sweden

\section{K.-E. Bergquist}

Department of Chemistry, Lund University, P.O. Box 124, 22100 Lund, Sweden

A. Idström · C. Olsson · T. Köhnke Bio-Based Fibres, Swerea IVF, P.O. Box 104, 43122 Mölndal, Sweden of the dissolution mechanism in which acetate ions form hydrogen bonds to cellulose and causes a diffuse solvation sheath of bulky tetrabutylammonium counterions to form. In turn, this leads to a steric repulsion that helps to keep the cellulose chains apart. Structural similarities to previously investigated cellulose solutions in aqueous tetrabutylammonium hydroxide were revealed by SAXS measurement. To what extent this corresponds to similarities in dissolution mechanism is discussed.

Keywords Cellulose dissolution - Ionic liquids · Nuclear magnetic resonance - Small angle X-ray scattering $\cdot$ Molecular dynamics simulations

\section{Introduction}

Cellulose is the most abundant natural polymer on Earth and an important raw material in a bio-based economy. As cellulose cannot be melted without chemical derivatization, it needs to be dissolved in order to be shaped into, e.g., fibres, films, or foams. Cellulose is insoluble in most common solvents, including water. Over the years, however, several classes of efficient cellulose solvents from wildly different chemical families have been discovered. Prominent among these are N-methylmorpholine-Noxide, lithium chloride in dimethylacetamide, various 
transition metal complexes in water, and superphosphoric acid (Liebert et al. 2010). A class of solvents that have attracted much interest during recent years is ionic liquids (IL) (Swatloski et al. 2002; Zhang et al. 2017). Some IL can dissolve technically relevant amounts of cellulose, and have also been used successfully in wood component separation (Kilpeläinen et al. 2007), chemical derivatization of cellulose (Gericke et al. 2012; Heinze and Gericke 2014), or in regeneration and cellulose fibre spinning (Sixta et al. 2015). However, imidazolium based IL, a family that includes several of the most-studied IL cellulose solvents, have been shown to be reactive towards reducing sugars, including the reducing terminus of cellulose (Gericke et al. 2012; Clough et al. 2015).

Alkylammonium IL have shown good potential in terms of cellulose dissolution capacity, stability and recyclability. Among these, tetrabutylammonium acetate (TBAAc) solutions in DMSO dissolve cellulose of high degree of polymerization (DP) in concentrations suitable for fibre spinning close to room temperature (Miao et al. 2014; Sun et al. 2015). As TBAAc has a melting point at the very upper edge of the IL range, the use of co-solvent is necessary to allow cellulose dissolution near room temperature. This is not necessarily a disadvantage; addition of dimethyl sulfoxide (DMSO) to 1-ethyl-3-methylimidazolium acetate (EmimAc) has previously been shown to promote the dissolution of cellulose $(\mathrm{Li}$ et al. 2016). For 1-allyl-3-methylimidazolium chloride, addition of low concentrations of DMSO enhances the dissolution of cellulose by weakening the electrostatic ion-ion interactions (Zhang et al. 2016). However, a further increase in DMSO concentration was found to severely decrease the dissolution capability. The dissolution mechanism has been investigated by nuclear magnetic resonance (NMR) techniques (Huang et al. 2016). In common with other IL cellulose solvent systems (Zhao et al. 2013; Payal et al. 2015), it was found to be crucially dependent on the hydrogen bonding ability of the anion. The cation was suggested to form ion-pairs with acetate at higher TBAAc concentrations (Huang et al. 2016), with detrimental effect on cellulose dissolution.

In this work, we map the dependence of the cellulose solubility on the concentration of TBAAc and investigate the dynamics and structure of the resulting cellulose solutions using diffusion NMR and small-angle X-ray scattering (SAXS). The interpretation of the experimental results is aided by molecular dynamics (MD) simulations, which provide mechanistic understanding of the system in molecular detail. Finally, we discuss the dissolution mechanism in comparison to that in aqueous "onium" hydroxides, a class of cellulose solvents that has attracted recent attention (Ema et al. 2014; Abe et al. 2015). We have recently investigated cellulose solutions in the tetrabutylammonium hydroxide (TBAH)/water solvent system using similar methods (Behrens et al. 2016; Gubitosi et al. 2016; Gentile and Olsson 2016).

\section{Experimental}

\section{Materials}

TBAAc, 97 wt.\% (lot no SHBF8128V), was acquired from Sigma Aldrich and extra dry DMSO, $99.7+$ wt.\% (lot no 1371030) was acquired from Fisher Scientific. MCC Avicel PH-101 was acquired from Food Machinery Corporation and dried in oven at $40^{\circ} \mathrm{C}$ for three hours before dissolution. The structures of all solvent components are shown in Fig. 1. Three TBAAc/DMSO stock solutions (1:8, 2:7, and 3:6 TBAAc:DMSO weight ratios) were first prepared by adding TBAAc to dry DMSO under stirring at $60^{\circ} \mathrm{C}$. The stock solutions were divided into separate samples and the required amount of cellulose was added under stirring. Overhead stirring was applied until the dissolution process reached steady state and the samples were thereafter removed and stored in room temperature.

\section{Light microscopy}

Light micrographs were captured using a Nikon SMZ1500 equipped with a 3.0MP CMOS microscope camera. The material was placed between two glass slides separated by

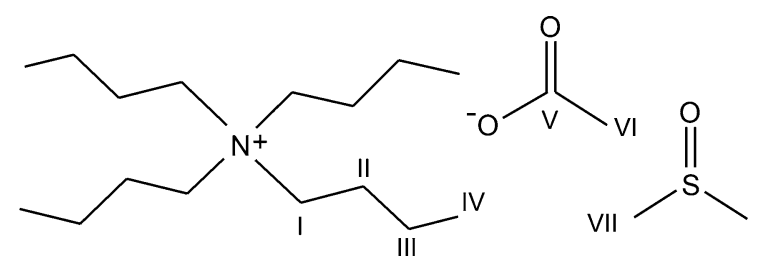

Fig. 1 Molecular structures of all components of the solvent, with all distinguishable carbon atoms labeled: $\mathrm{TBA}^{+}$(I, II, III, IV), acetate (V, VI), and DMSO (VII) 
a fixed distance to obtain comparable images of samples with dissolved and undissolved cellulose.

Turbidity measurements

Absorbance of solutions was measured at 800, 825, and $850 \mathrm{~nm}$ wavelengths on an UV/vis spectrometer (Specorde 200 Plus, Analytic Jena). None of the chemical species in the solution show any absorption at these wavelengths. Hence, measured absorbance is due to scattering and can be used to calculate the turbidity as $-\frac{1}{L} \ln (T)$, where $T$ is the average transmission from the three wavelengths and $L$ is the optical path length of the cuvette.

\section{${ }^{1} \mathrm{H}$ and ${ }^{13} \mathrm{C}$ NMR}

${ }^{1} \mathrm{H}$ and ${ }^{13} \mathrm{C}$ NMR measurements were performed on an 11.75 T Bruker Avance500 instrument equipped with a $5 \mathrm{~mm}$ broad band probe. All measurements were conducted at $40.00{ }^{\circ} \mathrm{C}$ and before each measurement the temperature was allowed to equilibrate for $5 \mathrm{~min}$. Deuterated trimethylsilyl propanoic acid $\left(\right.$ TMSP- $\left._{4}\right)$ in $\mathrm{D}_{2} \mathrm{O}$ was used as reference in a separate capillary inside each sample. ${ }^{1} \mathrm{H}$ NMR measurements were conducted using a $10.0 \mu$ s pulse and 1 scan. ${ }^{13} \mathrm{C}$ NMR measurements were conducted using a $9.2 \mu$ s pulse, up to 360 scans, and with a $0.2 \mathrm{~s}$ relaxation delay. All NMR spectra were processed with MestreNova 8.1.

The quantity of main interest is the difference in chemical shift relative to cellulose-free solutions, $\Delta \delta$. To compensate for non-specific medium effects when calculating $\Delta \delta$, the average of the three shift differences for the (hydrogen atoms bonded to) $\mathrm{TBA}^{+}$ carbon atoms II, III, and IV, for which the shift changes were small and mutually similar, was subtracted from $\Delta \delta$ for each solution.

\section{Diffusion NMR}

Diffusion NMR measurements were performed on a $4.68 \mathrm{~T}$ Bruker 200 instrument equipped with a commercial diffusion probe (DIF-25 $5 \mathrm{~mm}$ ). Measurements were conducted at $40^{\circ} \mathrm{C}$ using a pulsed field gradient stimulated spin-echo (PGSTE) pulse sequence. Measurements were conducted using a $9.5 \mu \mathrm{s}{ }^{1} \mathrm{H}$ pulse, 8 scans, and a $4.0 \mathrm{~s}$ relaxation delay. In the PGSTE a pair of trapezoidal narrow magnetic field gradient pulses were applied with amplitude $g$ of $52.11 \mathrm{G} / \mathrm{cm}$ and duration, $\tau$, of $2 \mathrm{~ms}$, encoded for spin displacement over a controlled observation time $\Delta$ of $140 \mathrm{~ms}$. Three $\pi / 2$ radio frequency pulses were applied in order to avoid the contribution of the spinspin relaxation time, $T_{2}$. The time between the first and second pulse was short $(3.2 \mathrm{~ms})$ while the time between the second and third pulse was longer (26.8 ms). The resulting spin-echo decays for $\mathrm{TBA}^{+}$ and DMSO were analyzed according to (Stejskal and Tanner 1965),

$\ln \left(\frac{I}{I_{0}}\right)=-D\left[(\gamma \tau g)^{2}\left(\Delta-\frac{\tau}{3}\right)\right]=-D b$

where $I$ is the echo amplitude, $I_{0}$ the amplitude at $g=0$, $\gamma$ the gyro-magnetic ratio of the proton, and $b$ is the so called $b$-factor (Le Bihan et al. 1986), reflecting the strength and timing of the gradients used to generate diffusion-weighted images. The spin-echo decay for the acetate was analyzed in a slightly different manner since one of the NMR peaks of the $\mathrm{TBA}^{+}$was overlapping with the only signal of the acetate. This resulted in a double exponential echo-decay, due to the two components. Since the diffusion rate of the TBA ${ }^{+}$ $\left(D_{T B A^{+}}\right)$could be extracted from other, non-overlapping, signals and the proportion, $p$, between the $\mathrm{TBA}^{+}$ and acetate was known, $p=1-p=0.5$, the echodecay of the two components could be fitted using

$\frac{I}{I_{0}}=p e^{-D_{T B A^{+}} b}+(1-p) e^{-D_{A c^{-}} b}$

giving the acetate diffusion rate. To validate the results, selected samples at low and high cellulose concentration were measured on an $11.75 \mathrm{~T}$ Bruker Avance500 equipped with an imaging probe. Due to the higher field, and the fact that at high and low concentrations the signals were less overlapping, it was possible to observe two resolved signals for the $\mathrm{TBA}^{+}$and acetate and consequently to measure the diffusion coefficients separately using Eq. 1. The results were in agreement with those obtained using Eq. 2.

\section{SAXS}

SAXS measurements were performed at the MAX II SAXS beamline I911-4 at MAX IV Laboratory in Lund, Sweden (Labrador et al. 2013). The scattered intensity (wavelength $=0.91 \AA$ ), was recorded in the scattering 
vector range $0.1-5.5 \mathrm{~nm}^{-1}$. The data was brought to absolute scale using water as a primary standard.

Molecular dynamics simulations

Systems composed of between zero and twelve cellodecaose molecules (referred to as 'cellulose' below) as well as 50, 90, or 120 TBAAc ion pairs and 926, 1215, or 1543 DMSO molecules, respectively, were considered. This gave solvent compositions of 1:8, 2:7, and 3:6 TBAAc:DMSO by weight. Simulations with zero, two, three, and five cellulose molecules were carried out in 1:8 TBAAc:DMSO solvent; with zero, one, two, three, six, and nine cellulose molecules in 2:7 TBAAc:DMSO solvent; and with zero, one, two, three, six, nine, and twelve cellulose molecules in 3:6 TBAAc:DMSO solvent. These compositions cover the concentration range in which cellulose is soluble. The degree of polymerization (DP) was at least one order of magnitude lower than what is typical in wood pulp, but should be adequate for the short-range structure to be reproduced. The cut-off for short-range interactions was $0.8 \mathrm{~nm}$. Long-range electrostatics were handled using the particle-mesh Ewald method (Essmann et al. 1995). The temperature was kept at $40{ }^{\circ} \mathrm{C}$ using the Bussi thermostat and the pressure at 1 bar using a weak coupling barostat (Bussi et al. 2007; Berendsen et al. 1984). All bond lengths were constrained to their equilibrium value using the LINCS algorithm (Hess et al. 1997). The time step was 2 fs and the total simulation time was between 300 and $400 \mathrm{~ns}$, of which the first $50 \mathrm{~ns}$ were discarded as equilibration. All calculations were performed using GROMACS version 4.6.3 (Hess et al. 2008).

The cellulose was modeled using the GLYCAM06 force field (Kirschner et al. 2008; Tessier et al. 2008). Bonded and Lennard-Jones parameters for acetate and $\mathrm{TBA}^{+}$were taken from the same force-field. Partial charges of $0,0.1,0.05,0.025$ and 0.0125 unit charges were assigned for the $\mathrm{TBA}^{+}$nitrogen and $\mathrm{TBA}^{+}$ carbons I, II, II, and IV, respectively. These charges where selected by analogy to shorter-chained tetraalkyl ammonium ions (Heyda et al. 2010), which follow the trend that the total charge of a methylene/ methyl group is approximately halved for each 'step' away from the nitrogen. Note that these partial charges give a total charge of 0.75 unit charges. This scaling- down of ionic charges is appropriate to compensate for the lack of electronic polarization in the model, which would otherwise cause the electrostatic interactions to be overestimated (Leontyev and Stuchebrukhov 2011). The acetate partial charges were taken from (Liu et al. 2010) and scaled by a factor 0.75. For DMSO the GAFF force-field for bonded and LennardJones interactions with partial charges from (Geerke et al. 2004). These partial charges were obtained by fitting to reproduce condensed-phase properties and their values reflect the polarization environment in the liquid state. Therefore, no scaling of the partial charges on DMSO molecules was applied.

Density functional theory

The chemical shift changes of acetate due to hydrogen bonding to cellulose were computed using density functional theory (DFT) calculations. Five hundred snapshots of acetate ions with at least one hydrogen bond to cellulose were extracted from the MD simulations. The conventional geometric criterion of an $\mathrm{O}-\mathrm{O}$ distance less than $0.35 \mathrm{~nm}$ and an $\mathrm{H}$-donor $\mathrm{O}$-acceptor $\mathrm{O}$ angle of less than 30 degrees was used. Carbon atoms bonded to hydrogendonating hydroxyl groups were cut out of the carbohydrate backbone and hydrogen atoms were added to satisfy the valence. This produced either methanol or ethylene glycol, the latter when adjacent hydroxyl groups were simultaneously hydrogen bonded to the same acetate ion. The structures were relaxed to the nearest energy minimum by geometry optimization using DFT with the B97-2 functional (Wilson et al. 2001), and the 6-311++g(d,p) basis set (McLean and Chandler 1980; Krishnan et al. 1980). NMR chemical shifts were calculated with the gaugeindependent atomic orbitals (GIAO) method using the same density functional with the pcSseg- 2 basis set (Jensen 2015), both of which are optimized for chemical shift calculations. In all calculations, a polarizable continuum solvent model corresponding to DMSO was included using the SCRF method. The calculations were performed using Gaussian09 (Frisch et al. 2009).

\section{Results}

Cellulose solubility

Micrographs and turbidity data are shown in Fig. 2. For the 1:8, 2:7, and 3:6 TBAAc:DMSO weight ratios, 
the turbidity starts deviating from zero around 6,10 , and 15 wt.\% of added MCC, respectively, Fig. 2a. Undissolved material appears in the micrographs around the same concentrations, Fig. 2c. The solubility limit corresponds approximately to a molar ratio of 1:1 between cellulose anhydroglucose units (AGU) and TBAAc. This is illustrated in Fig. 2b; the turbidity is close to zero up to an AGU/TBAAc molar ratio, $n_{A G U} / n_{T B A A c}$, of unity for all solvent compositions. Thus, there is a near-stoichiometric relationship such that each TBAAc formula unit is able to dissolve an amount of cellulose corresponding to one AGU. Cellulose solubility corresponding to a molar ratio 0.48:1 AGU:IL have been reported for several neat acetate IL (Wang et al. 2012).

In an earlier investigation (Huang et al. 2016), performed at room temperature, the maximum cellulose solubility was observed for $15 \mathrm{wt} \%$ TBAAc in DMSO (1.35:7.65 TBAAc:DMSO weight ratio); further increase in TBAAc concentration resulted in lower cellulose solubility. This feature was ascribed to an onset of ion-pairing above that concentration. In
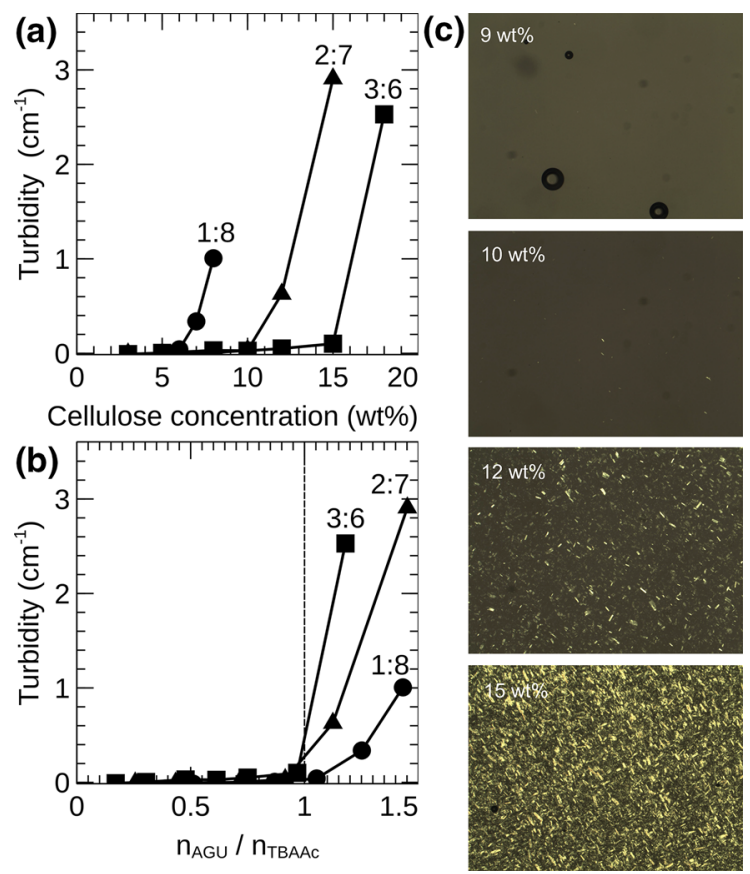

Fig. 2 Turbidity of the 1:8 (circles), 2:7 (triangles), and 3:6 (squares) TBAAc:DMSO samples as a function of cellulose concentration, expressed as: a wt.\% and b AGU/acetate molar ratio. c Light microscopy images of the 2:7 TBAAc:DMSO samples with $9,10,12$, and 15 wt.\% cellulose contrats, we did not observe a maximum in cellulose solubility at $40^{\circ} \mathrm{C}$. While there is no contradiction due to the difference in temperature, the difference in behavior at the two temperatures is somewhat surprising. Ion-pairing can generally be modelled as an equilibrium between free and paired ions and should thus show a gradual, rather than sudden, onset with changes in concentration and temperature. The observation that the border to the ion-paired stage does seem sharp suggests that there are aspects of this system that are not compeltely understood. Some tetrabutylammonium salts display liquid-liquid phase coexistance in aqueous solution (Weingärtner 2008). If some similar phenomenon occurs for TBAAc in DMSO solution, it may provide an alternative explanation for the observed facts.

\section{Acetate binding}

The simulation snapshots in Fig. 3a show that the acetate ions are predominantly bound to cellulose while $\mathrm{TBA}^{+}$ions occupy the space between the chains. The density maps in Fig. $3 b$ show that the acetate binding is localized to a handful of sites where the ions can form dual hydrogen bonds to cellulose hydroxyl groups. Neither the DMSO solvent nor $\mathrm{TBA}^{+}$ions show the same type of highly localized binding but rather form loose solvation sheaths around the cellulose chains.

We performed NMR experiments to quantify the binding of acetate to cellulose, analogous to those presented in (Huang et al. 2016) but directly on dissolved cellulose, rather than on the model compound cellobiose. The ${ }^{1} \mathrm{H}$ and ${ }^{13} \mathrm{C}$ NMR results are shown in Fig. 4a, b, respectively, in terms of $\Delta \delta$, the difference in chemical shift relative to cellulose-free solution. See SI for example spectra. For each of the acetate nuclei, there is a near-linear change in $\Delta \delta$ with cellulose concentration up to the cellulose solubility limit. $\Delta \delta$ for methyl hydrogen and for carbon $\mathrm{V}$ increases with increasing cellulose concentration. There is a corresponding decrease in $\Delta \delta$ for carbon VI. With the exception of the ${ }^{1} \mathrm{H} \Delta \delta$ from the TBA ${ }^{+}$ carbon I, only small shift changes can be seen for $\mathrm{TBA}^{+}$and DMSO. The TBA ${ }^{+1} \mathrm{H} \Delta \delta$ depends mainly on the cellulose concentration, rather than the solvent composition, and therefore seems to originate from interaction with cellulose. 
(a)

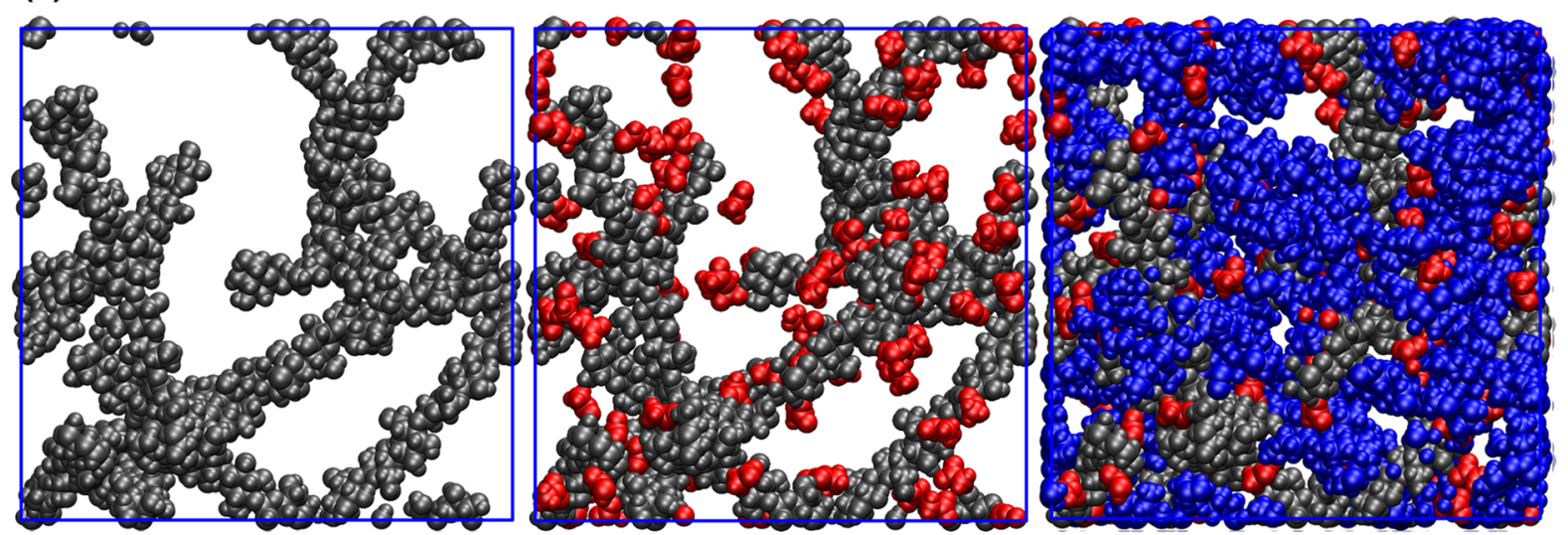

(b)

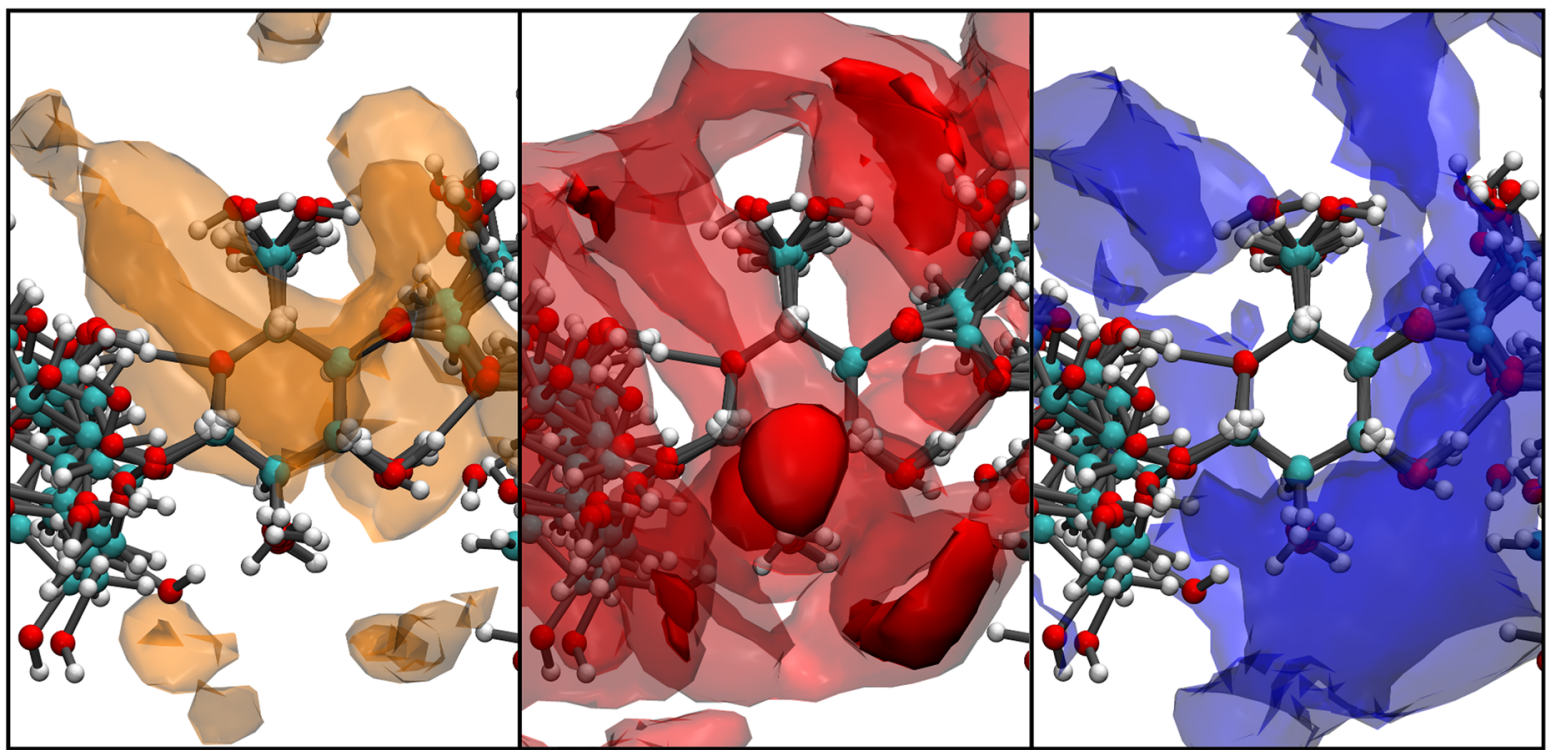

Fig. 3 a Visualization of one frame of the simulation trajectory of the 2:7 TBAAc:DMSO solvent with 10 wt.\% cellulose. From left to right cellulose (gray); cellulose and acetate ( $r e d)$; and cellulose, acetate, and $\mathrm{TBA}^{+}$(blue). b Density maps, i.e., isosurfaces of the concentration field around a cellulose AGU for DMSO sulfur

The DFT calculations yielded average differences in shift between the free and hydrogen bonded states of $0.079 \pm 0.002 \mathrm{ppm}$ for the acetate ${ }^{1} \mathrm{H}$ signal. For the ${ }^{13} \mathrm{C}$ signals, DFT gave shift differences of $1.39 \pm 0.09$ and $-0.50 \pm 0.03 \mathrm{ppm}$ for acetate carbon atoms $\mathrm{V}$ and VI, respectively. The magnitudes of the two ${ }^{13} \mathrm{C}$ shift differences were thus underestimated as compared to the maximum observed $\Delta \delta$. However, the signs and the approximate relative magnitudes of the ${ }^{13} \mathrm{C}$ shift differences are consistent with experiment. This lends strong support to the interpretation that the (orange), acetate carboxyl carbon (red) and $\mathrm{TBA}^{+}$nitrogen (blue). The transparent surfaces encloses regions with local concentration at least double the bulk concentration and the opaque surfaces (present only for acetate) correspond to twenty times the bulk concentration. (Color figure online)

acetate shift changes are being due to hydrogen bonding, as suggested in an earlier investigation (Huang et al. 2016).

We estimate the proportion of bound acetate as from the shift changes of acetate carbon atoms through

$$
P_{A c^{-}}=\frac{\Delta \delta^{\max }-\Delta \delta}{\Delta \delta^{\max }},
$$

where $\Delta \delta^{\max }$ is the shift change for the maximum cellulose concentration. In Fig. 4c, we compare this estimate to the amount of bound acetate as a function 

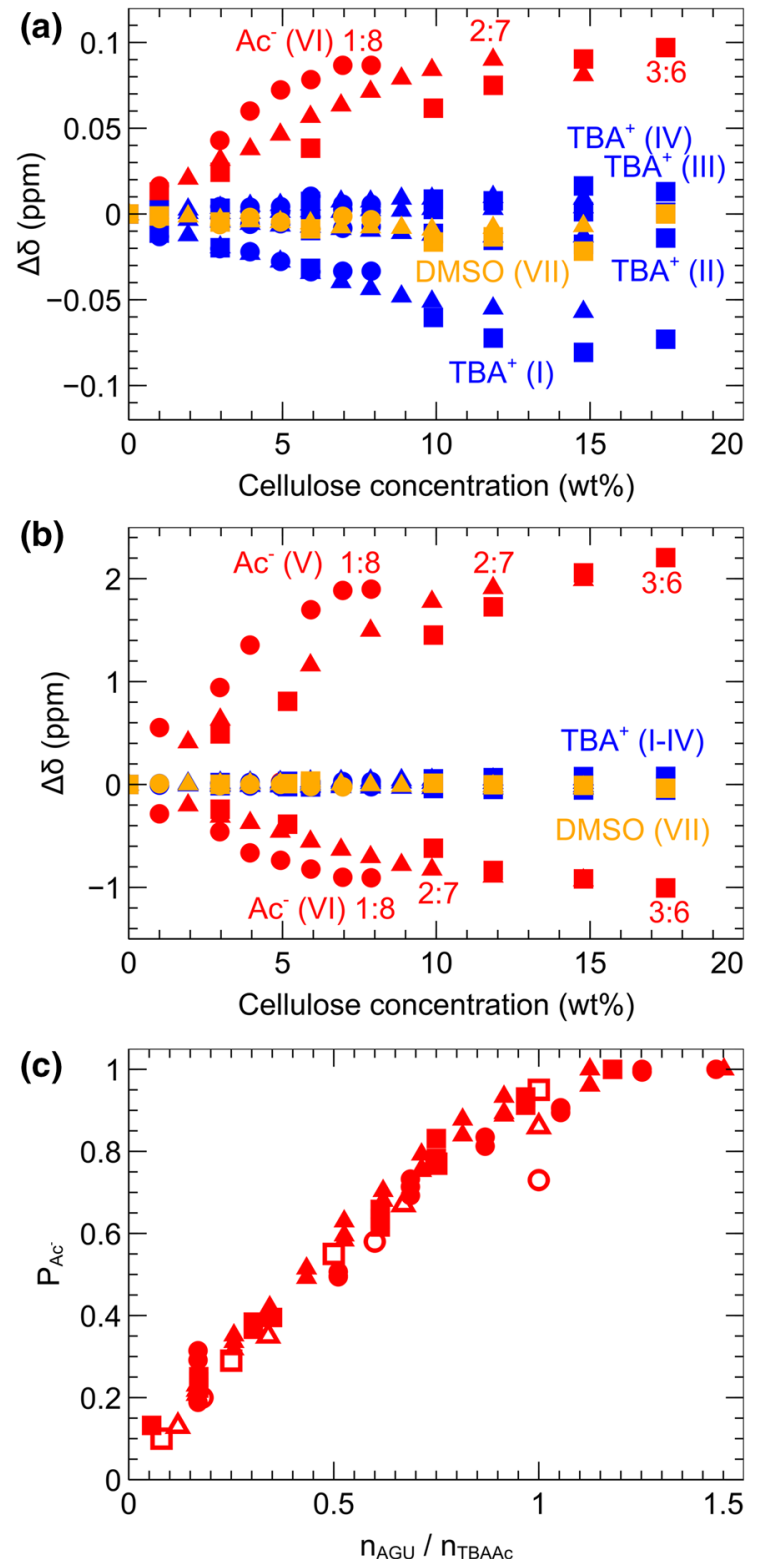

Fig. $4{ }^{1} \mathrm{H}$ (a) and ${ }^{13} \mathrm{C}$ (b) NMR chemical shift changes for the atoms indicated, see Fig. 1, in acetate $($ red $), \mathrm{TBA}^{+}$(blue) and DMSO (orange) in 1:8 (circles), 2:7 (triangles), and 3:6 (squares) TBAAc:DMSO weight ratio solvent, as a function of cellulose concentration. c The fraction of acetate bound to cellulose obtained from the NMR chemical shifts via Eq. 3 (filled symbols) and from simulation (open symbols), see text, for 1:8 (circles), 2:7 (triangles), and 3:6 (squares) TBAAc:DMSO ratios. (Color figure online)

of $n_{A G U} / n_{T B A A c}$ with the proportion of bound acetate calculated from the simulation trajectories. For this analysis, acetate ions with their carboxyl carbon atoms within $0.38 \mathrm{~nm}$ of any cellulose atom are considered bound to cellulose. This cut-off distance coincides with the minimum beyond the first peak of the corresponding radial distribution function. Both the experimental and simulated data fall close to a line with a slope slightly below unity up to $n_{A G U} / n_{T B A A C}=1$, corresponding to the cellulose solubility limit. The agreement confirms that acetate ions binds to cellulose with a stoichiometry of approximately one per AGU, which appears to explain why the solubility follows that approximate stoichiometry.

\section{Diffusion}

Diffusion NMR spectroscopy was used to follow the diffusion coefficients of the solvent species as a function of cellulose concentration. The measured and simulated diffusion coefficients are shown in Fig. 5. In the absence of cellulose, the acetate ions diffuse faster than the $\mathrm{TBA}^{+}$ions. This is typical for diffusion of ions in solution but contrasts with the situation in neat IL, where large cations tend to diffuse faster than small anions. The diffusion rates of all components of the solvent decreases with increasing cellulose concentration, with the largest change seen for acetate. For the highest cellulose concentrations, the diffusion coefficients of acetate and $\mathrm{TBA}^{+}$almost exactly coincide. The simulations are in fair quantitative agreement with the experiments. As the force field has not been specifically parametrized to reproduce diffusion coefficients, this inspires confidence in its general reliability.

A recent study on cellulose dissolved in TBAH/water (Gentile and Olsson 2016), showed that a simple twostate model describes the binding of $\mathrm{TBA}^{+}$to cellulose in that solvent. This model assumes that the observed diffusion coefficient of each species $i$ is given by

$D_{i}=\left(1-P_{i}\right) D_{i}^{0}+P_{i} D_{\text {cell }}$,

where $P_{i}$ is the proportion of bound molecules of species $i$, c.f. Eq. 3. $D_{i}$ is the observed diffusion coefficient $D_{i}^{0}$ is the diffusion coefficient of a 'free' molecule of species $i$, assumed here to be equal to the diffusion coefficient in cellulose-free solution, and $D_{\text {cell }}$ is the diffusion coefficient of cellulose, and any molecules bound to cellulose. As $D_{\text {cell }} \approx 0$, Eq. 4 simplifies to $D_{i}=\left(1-P_{i}\right) D_{i}^{0}$. If species $i$ binds stoichiometrically to cellulose, 


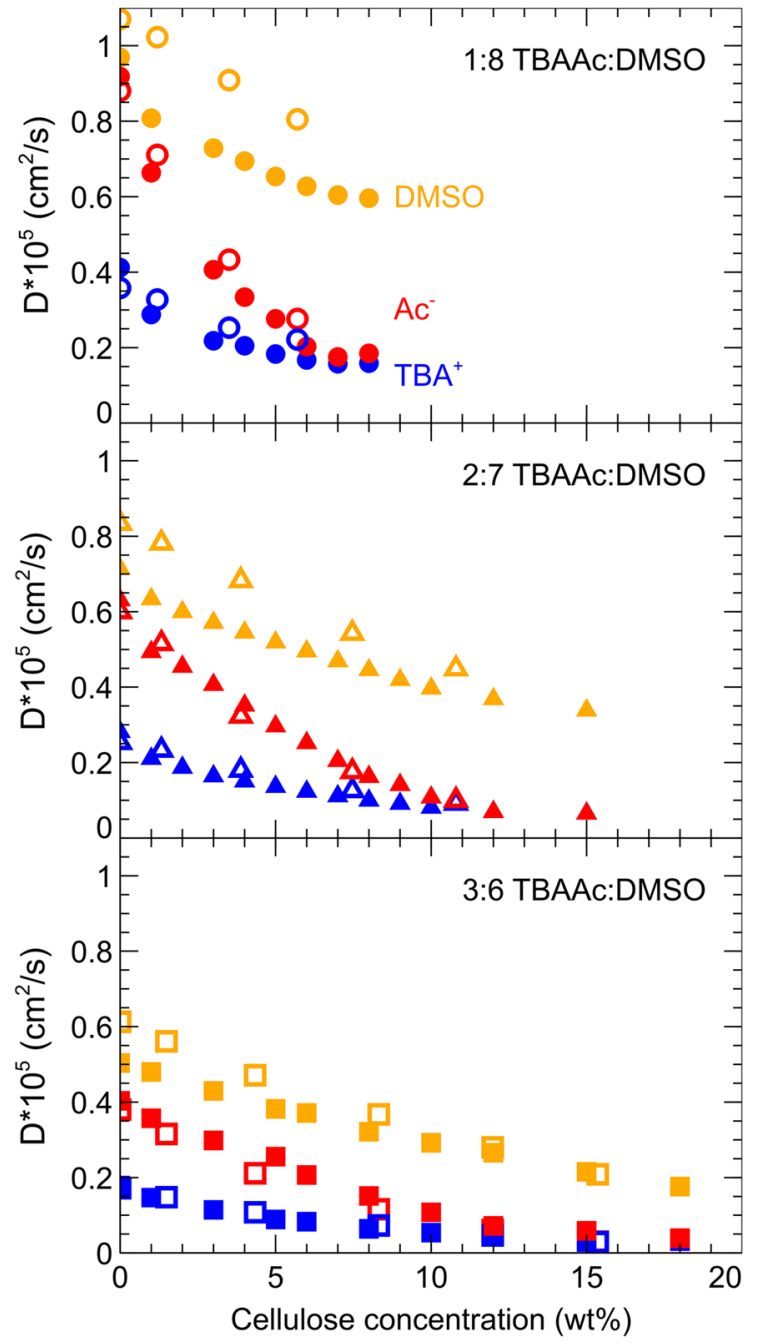

Fig. 5 Diffusion coefficients from NMR (filled symbols) and simulation (open symbols) for DMSO (orange), acetate (red), and $\mathrm{TBA}^{+}$(blue) for the TBAAc:DMSO weight ratios indicated. (Color figure online)

$P_{i}=N_{i} \frac{n_{A G U}}{n_{i}}$

where $n_{A G U}$ and $n_{i}$ are the number of moles of anhydroglucose units and species $i$, respectively, and $N_{i}$ is the coordination number for binding of species $i$ to cellulose.

The diffusion coefficients, normalized by their value for zero cellulose concentration, $D_{i}^{0}$, were plotted against the corresponding molar ratio $n_{A G U} / n_{i}$ in Fig. 6. If the two-state model holds, this should yield straight line with the slope $-N_{i}$ for each species. For acetate, the data for low cellulose concentrations falls close to, though slightly below,
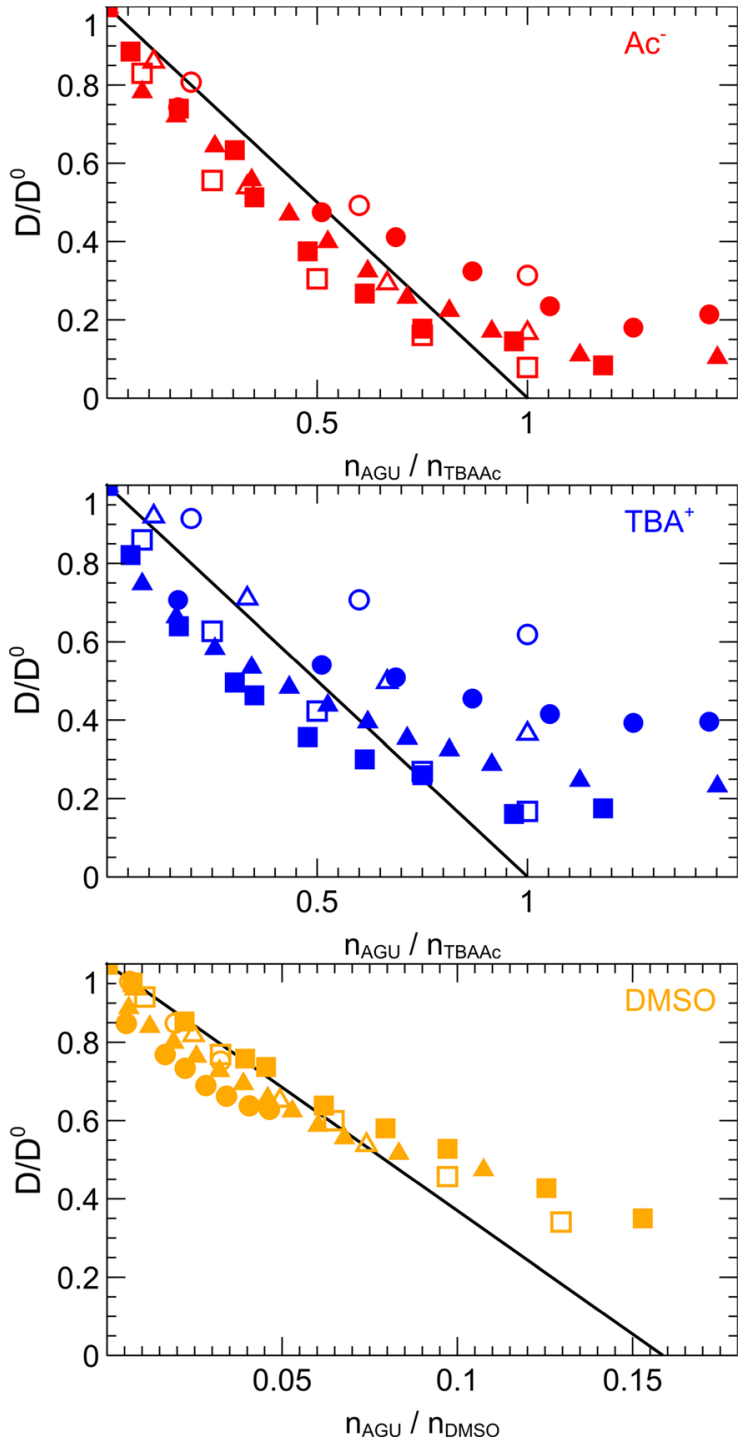

Fig. 6 Alternative representation of the data in Fig. 5 that allows interpretation in terms of the two-state model, see Eqs. 4 and 5. The diffusion coefficients are here normalized by the value for zero cellulose concentration and the cellulose concentration is given as the molar ratio $n_{A G U} / n_{T B A A c}$ or $n_{A G U} / n_{D M S O}$ as appropriate. Symbols corresponding to different TBAAc:DMSO weight ratios are distinguished by the choice of symbols: $1: 8$ by circles, $2: 7$ by triangles, and $3: 6$ by squares

the line expressing the expectation raised by Fig. 4c. Namely, that the two-state model holds with $N_{A c^{-}} \approx 1$. For $n_{A G U} / n_{T B A A C}$ greater than about 0.5 , the curves start to diverge. For $\mathrm{TBA}^{+}$, the curves diverge already for small cellulose concentrations. This leads to the conclusion that the two-state model is not able to describe the diffusion behavior of $\mathrm{TBA}^{+}$ions. The 
DMSO curves are approximately linear with a slope corresponding to $N_{D M S O}=6$ for small $n_{A G U} / n_{D M S O}$.

It appears that the two-state model can roughly describe the diffusion of acetate and DMSO for low cellulose concentration, but not that of $\mathrm{TBA}^{+}$for any appreciable cellulose concentration. This is in stark contrast to previous results for the $\mathrm{TBAH} /$ water system (Gentile and Olsson 2016). In that work, the solvent diffusion coefficient was almost unaffected by the presence of cellulose. (The hydroxide anion could not be observed separately as the signal from its exchangeable proton merges with the water signal.) What little dependence was observed, a few percent, could be accounted for by the excluded volume of the cellulose molecules (Jönsson et al. 1986). Here, in contrast, the diffusion coefficient deviates from the cellulose-free value by as much as tens of percent, which is incompatible with the excluded volume explanation.

To explore the dynamic correlations in the simulated system, we computed the life time of contacts between the various species. The contact life time, $t_{\text {life }}$, is a measure of the average duration of a contact and was calculated as described in SI. We considered the simulated systems containing one cellulose molecule each, so that the results are relevant in the limit of infinite dilution. For our purposes, molecules are in contact if their 'central' atoms are within $0.72 \mathrm{~nm}$ of each other. This cut-off distance is sufficient to accommodate contacts between the largest species in the system and was applied also to smaller species for consistency. For DMSO we take the sulfur atom to be central, for $\mathrm{TBA}^{+}$nitrogen, for acetate the carboxyl carbon and for cellulose the hydroxyl oxygen atom bonded to C2 (other choices for cellulose gave similar results).

Contact life times for selected pairs of species are shown in Fig. 7. For acetate-cellulose contacts, $t_{\text {life }}$, falls between 10 and $20 \mathrm{~ns}$, an order of magnitude more long lived than $\mathrm{TBA}^{+}$-acetate ion pairs and two orders of magnitude more long-lived than acetateDMSO contacts. The long life time of acetatecellulose contacts is consistent with the highly localized binding of acetate described above, see Fig. 3, and validates the assumptions in the two-state model that the diffusion coefficient of bound acetate is effectively that of cellulose. The life-times of for $\mathrm{TBA}^{+}$-cellulose contacts vary between 1 and $4 \mathrm{~ns}$,

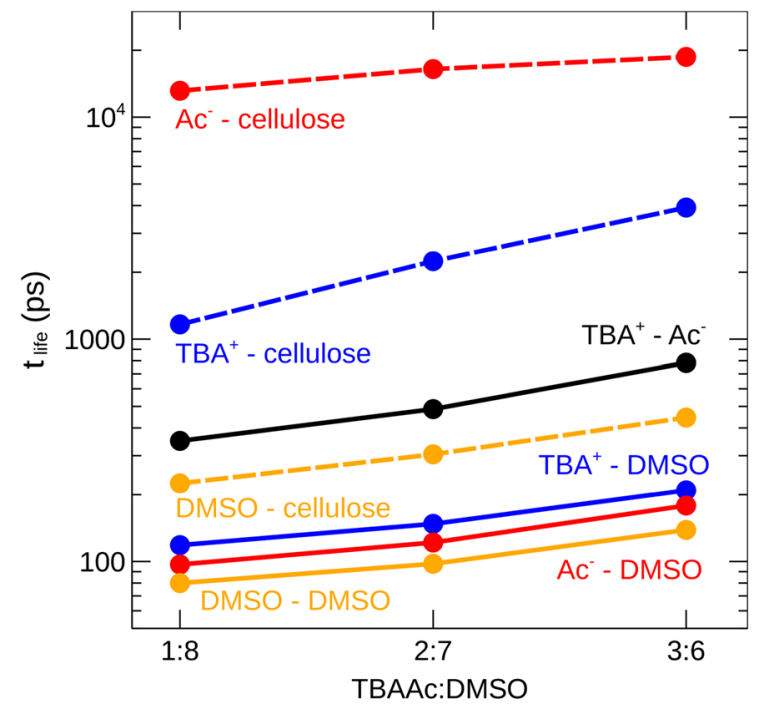

Fig. 7 Simulated contact life times, $t_{\text {life }}$, for the pairs of species indicated as a function of TBAAc:DMSO ratio for low cellulose concentration

about one order of magnitude longer than that of $\mathrm{TBA}^{+}-\mathrm{DMSO}$ contacts. The large slow-down can be rationalized by the strong electrostatic interaction between $\mathrm{TBA}^{+}$ions and cellulose chains with a high density of bound acetate. This may also explain the observation that the diffusion coefficients of $\mathrm{TBA}^{+}$ and acetate are approximately equal in solutions saturated with cellulose, see Fig. 5. DMSO-cellulose contacts are consistently about three times as long lived as DMSO-DMSO contacts. This provides a rationale why the two-state model seems to also describe the data for DMSO: while the DMSO molecules in the first cellulose solvation shell are not bound in the same sense as acetate, they a sufficiently slowed down for it to have a considerable effect on the diffusion coefficient.

\section{SAXS}

SAXS experiments were performed to probe the solution structure. Data obtained for $10 \mathrm{wt} \% \mathrm{MCC}$ in the 2:7 TBAAc: DMSO solvent are shown in Fig. 8. Measurements for lower cellulose concentrations were not possible due to low transmission, resulting from strong X-ray absorption by the DMSO sulfur. There is a hump in the vicinity of $q=3 \mathrm{~nm}^{-1}$. A similar feature exists in cellulose solutions in the TBAH/water system 
(Behrens et al. 2016). As we argued that study, it signifies the presence a core-shell structure.

We used The Pedersen-Schurtenberger model (PSM) to fit the data in the high- $q$ range (Pedersen and Schurtenberger 1996), red curve in Fig. 8a. This model describes individual semi-flexible polymer chains with excluded volume interactions. It is a discrete representation of the worm-like chain model of Kratky and Porod, applied in the pseudo-continuous limit. This model was implemented considering a nonhomogeneous radial scattering length density (SLD) distribution to describe the enhanced scattering around $q=3 \mathrm{~nm}^{-1}$, using the same approach as in ref. (Behrens et al. 2016). A core-shell cross section model was introduced, with the shell electron density being about $90 \%$ of the bulk solvents, as depicted in Fig. 8b. For lower $q$-values, the scattered intensity is significantly higher compared to what is predicted by the PSM. This shows that the cellulose molecules form aggregates, again similar to what was previously
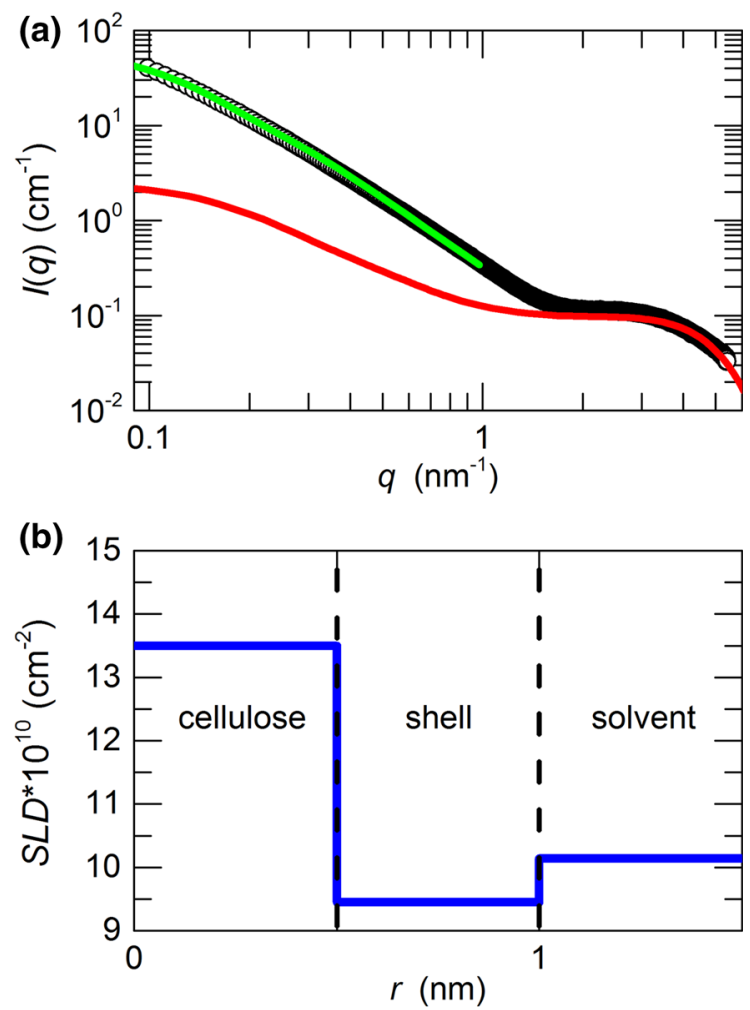

Fig. 8 SAXS profile in absolute intensity and calculated curves for the form factor of a single semi-flexible cellulose chain (red) and of a fractal cluster (green) for $10 \mathrm{wt} \% \mathrm{MCC}$ in 2:7 TBAAc:DMSO. b SLD radial profile used for the calculation of the form factor of the single chain. (Color figure online) observed in aqueous tetrabutylammonium hydroxide solvent (Behrens et al. 2016; Gubitosi et al. 2016). The green curve in Fig. $8 \mathrm{a}$ was obtained by modelling the aggregates as fractal clusters with a radius of gyration of $14 \mathrm{~nm}$ and a fractal dimension $d=2.4$, see SI for details (Hammouda 2010). Hence, the aggregates appear somewhat more dense than, for example, a single excluded-volume polymer coil, for which $d \approx 5 / 3$.

We present the simulated radial SLD profile and the corresponding profiles of the contributions from the various chemical species in Fig. 9. The profiles are computed from the atomic number density in cylindrical shells around the C1-C4 axis in each AGU. The distance from this axis is denoted $r$. The atoms are assumed to be point scatterers and we therefore do not expect quantitative accuracy. Acetate is strongly enriched in the region around $r=0.5 \mathrm{~nm}$, but depleted

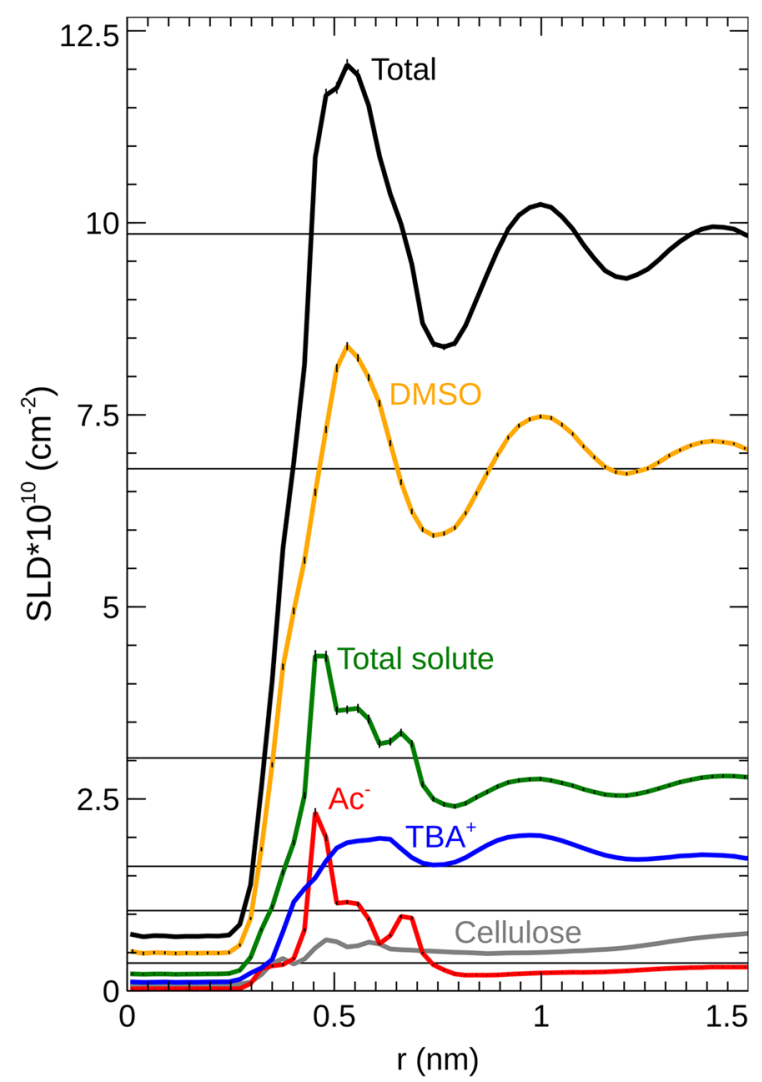

Fig. 9 SLD profile of cellulose solvation sheath, not including the contribution from the cellulose chain itself, calculated from simulation and the contributions from individual species to the SLD profile, as indicated. "Cellulose" referrs to the contribution from cellulose chains other than the central one 
for larger $r$. TBA ${ }^{+}$is enriched to varying degree in the entire region between $r=0.5$ and $1.5 \mathrm{~nm}$. This distribution of ions around the cellulose chain can be described as an 'ionic atmosphere', with the inner part consisting of bound acetate ions and the outer part consisting of $\mathrm{TBA}^{+}$counterions. Other cellulose molecules are excluded from the region in which $\mathrm{TBA}^{+}$is enriched due to steric exclusion by the bulky ions. The total contribution from solute species gives a deficit of SLD in the region around a cellulose molecule compared to bulk solution. The DMSO solvent has the wavy structure that characteristic of a dense fluid and reflects the bulk packing of solvent molecules (Hansen and McDonald 2006). The length scale of this structural feature corresponds to $q$ above the experimental range and is therefore of limited relevance for interpretation of the SAXS data. In the total SLD profile, the same wavy structure can be seen but beyond the first peak, the curve is depressed such that the troughs are deeper than the peaks are high. Thus, the simulations qualitatively reproduce the presence of a low-SLD shell around the each cellulose molecule and suggest that it is indicative of repulsion between cellulose molecules.

\section{Discussion and conclusions}

The results above are all consistent with a mechanistic picture where approximately one acetate ion binds to each AGU by strong, long-lived hydrogen bonds. This gives the cellulose an effective negative charge that causes $\mathrm{TBA}^{+}$counterions to form a sheath around each cellulose chain. The electrostatic and steric repulsion between bound $\mathrm{TBA}^{+}$cations appears to prevent association between cellulose molecules and favor a molecularly dissolved state. Hydrogen bonds to anions are particularly strong because the iondipole interaction is favorable. The efficiency of ionic liquids incorporating strong hydrogen bond acceptor anions was observed already in the pioneering work on ILs (Swatloski et al. 2002), and further discussed by Sellin et al. (Sellin et al. 2010). This being the driving force suggests that the solvents ability to dissolve cellulose is strongly decreased if water or other hydrogen bond donors, e.g. alcohols, are present as impurities, competing with cellulose for hydrogen bonding with the acetate ion. This is indeed observed experimentally; the presence of water decreases cellulose solubility in IL solvents (Olsson et al. 2014; Le et al. 2012). A necessary feature of the solvent for the hydrogen bonding mechanism thus appears to be the frustration in the solvent that contains a strong hydrogen bond acceptor, the acetate ion, but no donor. The addition of water or alcohol relaxes the frustration by allowing the formation of more hydrogen bonds to acetate. The need for a system to be frustrated to be a good cellulose solvent probably applies generally to IL solvents, for instance, a similar concepts has been applied to explain the difference in cellulose-dissolving ability of EmimCl and $\mathrm{BmimCl}$ by ( $\mathrm{Li}$ et al. 2015), and also to amine oxides like N-Methylmorpholine-N-oxide (NMMO) (Fink et al. 2001). However, in aqueous systems, where abundant opportunity generally exists to form both acceptor and donor hydrogen bonds, frustrated conditions can only be attained for extremely high solute concentrations, such as in molten salt hydrates (Heinze and Koschella 2005).

There are structural similarities between cellulose solutions in the TBAAc/DMSO system and in aqueous TBAH, c.f., Fig. 8 and previously published scattering curves for the TBAH/water system (Behrens et al. 2016; Gubitosi et al. 2016). Essentially, the solvation sheath of $\mathrm{TBA}^{+}$ions around the cellulose molecules is found in both systems, which indicates the presence of charge on the cellulose chains. This should favor molecular dissolution as $\mathrm{TBA}^{+}$, unlike small cations, is not likely to be sterically compatible with cellulose aggregation. Indeed, alkali cations poison the cellulose-dissolving ability of the TBAH/water system, which can be restored by adding a crown ether (Ema et al. 2014). A naive analogy to the acetate binding observed here would suggest that the mechanism of charging in aqueous TBAH is binding of $\mathrm{OH}^{-}$ions. However, in light of the lack of frustration in aqueous systems (Medronho et al. 2012), this appears unlikely. An alternative explanation for the presence of charge on the cellulose chains is provided by the recent observation that cellulose is deprotonated in aqueous alkali (Bialik et al. 2016), as anticipated by Lindman et al. (Medronho and Lindman 2014; Alves et al. 2015). Thus, we argue that only the role of the $\mathrm{TBA}^{+}$ cation is similar between the TBAAc/DMSO and TBAH/water solvent system; the primary driving forces for cellulose dissolution are distinct. 
The TBAAc/DMSO cellulose solvent system appears to have favorable properties for cellulose shaping applications, e.g. fibre spinning. The presence of DMSO co-solvent dramatically lowers viscosity compared to neat IL and offers a way to tune this property. This may come at no cost in terms of maximum attainable cellulose concentration; as we have shown above, TBAAc in DMSO is mole for mole a more efficient cellulose solvent than many neat acetate-based IL. The cellulose solubility is, furthermore, predictable over an appreciable composition range thanks near-stoichiometric relationship found above, namely that one mole of TBAAc dissolves an amount of cellulose corresponding to approximately one mole of glucose residues.

Acknowledgments This research was performed with financial support from The Swedish Research Council Formas, the Bo Rydin Foundation, and Södra Skogsägarna Foundation for Research, Development and Education through the research program Avancell - Centre for Fibre Engineering.

Open Access This article is distributed under the terms of the Creative Commons Attribution 4.0 International License (http:// creativecommons.org/licenses/by/4.0/), which permits unrestricted use, distribution, and reproduction in any medium, provided you give appropriate credit to the original author(s) and the source, provide a link to the Creative Commons license, and indicate if changes were made.

\section{References}

Abe M, Kuroda K, Ohno H (2015) Maintenance-free cellulose solvents based on onium hydroxides. ACS Sustain Chem Eng 3:1771-1776

Alves L, Medronho BF, Antunes FE, Romano A, Miguel MG, Lindman B (2015) On the role of hydrophobic interactions in cellulose dissolution and regeneration: colloidal aggregates and molecular solutions. Colloids Surf A Physicochem Eng Asp 483:257-263

Behrens MA, Holdaway JA, Nosrati P, Olsson U (2016) On the dissolution state of cellulose in aqueous tetrabutylammonium hydroxide solutions. RSC Adv 6:30,199-30,204

Berendsen HJC, Postma JPM, van Gunsteren WF, DiNola A, Haak JR (1984) Molecular dynamics with coupling to an external bath. J Chem Phys 81:3684-3690

Bialik E, Stenqvist B, Fang Y, Östlund Å, Furó I, Lindman B, Lund M, Bernin D (2016) Ionization of cellobiose in aqueous alkali and the mechanism of cellulose dissolution. J Phys Chem Lett 7:5044-5048

Bussi G, Donadio D, Parrinello M (2007) Canonical sampling through velocity rescaling. J Chem Phys 126(014):101
Clough MT, Geyer K, Hunt PA, Son S, Vagt U, Welton T (2015) Ionic liquids: not always innocent solvents for cellulose. Green Chem 17:231-243

Ema T, Komiyama T, Sunami S, Sakai T (2014) Synergistic effect of quaternary ammonium hydroxide and crown ether on the rapid and clear dissolution of cellulose at room temperature. RSC Adv 4:2523-2525

Essmann U, Perera L, Berkowitz ML, Darden T, Lee H, Pedersen LG (1995) A smooth particle mesh Ewald method. J Chem Phys 103:8577-8592

Fink H, Weigel P, Purz HJ, Ganster J (2001) Structure formation of regenerated cellulose materials from NMMO-solutions. Prog Polym Sci 26:1473-1524

Frisch MJ, Trucks GW, Schlegel HB, Scuseria GE, Robb MA, Cheeseman JR, Scalmani G, Barone V, Mennucci B, Petersson GA, Nakatsuji H, Caricato M, Li X, Hratchian HP, Izmaylov AF, Bloino J, Zheng G, Sonnenberg JL, Hada M, Ehara M, Toyota K, Fukuda R, Hasegawa J, Ishida M, Nakajima T, Honda Y, Kitao O, Nakai H, Vreven T, Montgomery Jr JA, Peralta JE, Ogliaro F, Bearpark M, Heyd JJ, Brothers E, Kudin KN, Staroverov VN, Kobayashi R, Normand J, Raghavachari K, Rendell A, Burant JC, Iyengar SS, Tomasi J, Cossi M, Rega N, Millam JM, Klene M, Knox JE, Cross JB, Bakken V, Adamo C, Jaramillo J, Gomperts R, Stratmann RE, Yazyev O, Austin AJ, Cammi R, Pomelli C, Ochterski JW, Martin RL, Morokuma K, Zakrzewski VG, Voth GA, Salvador P, Dannenberg JJ, Dapprich S, Daniels AD, Farkas O, Foresman JB, Ortiz JV, Cioslowski J, Fox DJ (2009) Gaussian 09, Revission A.02

Geerke DP, Oostenbrink C, van der Vegt NFA, van Gunsteren WF (2004) An effective force field for molecular dynamics simulations of dimethyl sulfoxide and dimethyl sulfoxidewater mixtures. J Phys Chem B 108:1436-1445

Gentile L, Olsson U (2016) Cellulose-solvent interactions from self-diffusion NMR. Cellulose 23:2753-2758

Gericke M, Fardim P, Heinze T (2012) Ionic liquids-promising but challenging solvents for homogeneous derivatization of cellulose. Molecules 17:7458-7502

Gubitosi M, Duarte H, Gentile L, Olsson U, Medronho B (2016) On cellulose dissolution and aggregation in aqueous tetrabutylammonium hydroxide. Biomacromolecules $17: 2873-2881$

Hammouda B (2010) Analysis of the Beaucage model. J Appl Crystallogr 43:1474-1478

Hansen JP, McDonald IR (2006) Theory of simple liquids. Academic Press, London

Heinze T, Gericke M (2014) Ionic liquids as solvents for homogeneous derivatization of cellulose: challenges and opportunities. In: Fang Z, Smith RL Jr, Qi X (eds) Production of biofuels and chemicals with ionic liquids, vol 1 . Springer, Dordrecht, pp 107-144

Heinze T, Koschella A (2005) Solvents applied in the field of cellulose chemistry-a mini review. Polímeros: Ciêntas e Technologia 15:84-90

Hess B, Bekker H, Berendsen HJC, Fraaije JGEM (1997) Lincs: a linear constraints solver for molecular simulations. J Comput Chem 18:1463-1472

Hess B, Kutzner C, van der Spoel D, Lindahl E (2008) Gromacs 4: algorithms for highly efficient, load-balanced, and 
scalable molecular simulation. J Chem Theory Comput 4:435-447

Heyda J, Lund M, Ončák M, Slavíček P, Jungwirth P (2010) Reversal of hofmeister ordering for pairing of $\mathrm{nh}_{4}^{+}$vs alkylated ammonium cations with halide anions in water. J Phys Chem B 114:10,843-10,852

Huang Y, Xin P, Li J, Shao Y, Huang C, Pan H (2016) Roomtemperature dissolution and mechanistic investigation of cellulose in a tetra-butylammonium acetate/dimethyl sulfoxide system. ACS Sustain Chem Eng 4:2286-2294

Jensen F (2015) Segmented contracted basis sets optimized for nuclear magnetic shielding. J Chem Theory Comput 11:132-138

Jönsson B, Wennerström H, Nilsson PG, Linse P (1986) Selfdiffusion of small molecules in colloidal systems. Colloid Polym Sci 264:77-88

Kilpeläinen I, Xie H, King A, Granstrom M, Heikkinen S, Argyropoulos DS (2007) Dissolution of wood in ionic liquids. J Agric Food Chem 55:9142-9148

Kirschner KN, Yongye AB, Tschampel SM, Gonzalez-Outierino J, Daniels CR, Foley BL, Woods RJ (2008) Glycam06: a generalizable biomolecular force field. Carbohydrates. J Comput Chem 29:622-655

Krishnan R, Binkley JS, Seeger R, Pople JA (1980) Self consistent molecular orbital methods. XX. A basis set for correlated wave functions. J Chem Phys 72:650

Labrador A, Cerenius Y, Svensson C, Theodor K, Plivelic T (2013) The yellow mini-hutch for SAXS experiments at MAX IV laboratory. J Phys 425(072):019

Le KA, Sescousse R, Budtova T (2012) Influence of water on cellulose-emimac solution properties: a viscometric study. Cellulose 19:45-54

Le Bihan D, Breton E, Lallemand D, Grenier P, Cabanis E, Laval-Jeantet M (1986) Mr imaging of intravoxel incoherent motions: application to diffusion and perfusion in neurologic disorders. Radiology 161:401

Leontyev I, Stuchebrukhov A (2011) Accounting for electronic polarization in non-polarizable force fields. Phys Chem Chem Phys 13:2613-2626

Li Y, Liu X, Zhang S, Yao Y, Yao X, Xu J, Lu X (2015) Dissolving process of a cellulose bunch in ionic liquids: a molecular dynamics study. Phys Chem Chem Phys 17:17,894-17,905

Li X, Zhang Y, Tang J, Lan A, Yang Y, Gibril M, Yu M (2016) Efficient preparation of high concentration cellulose solution with complex DMSO/ILS solvent. J Polym Res 23:32

Liebert TF, Heinze TJ, Edgar KJ (eds) (2010) Cellulose solvents: for analysis, shaping and chemical modification. American Chemical Society, Washington, DC

Liu H, Sale KL, Holmes BM, Simmons BA, Singh S (2010) Understanding the interactions of cellulose with ionic liquids: a molecular dynamics study. J Phys Chem B 114:4293-4301

McLean AD, Chandler GS (1980) Contracted gaussian basis sets for molecular calculations. I. Second row atoms, $\mathrm{z}=$ 11-18. J Chem Phys 72:5639

Medronho B, Lindman B (2014) Competing forces during cellulose dissolution: from solvents to mechanisms. Curr Opin Colloid Interface Sci 19:32-40

Medronho B, Romano A, Miguel MG, Stigsson L, Lindman B (2012) Rationalizing cellulose (in)solubility: reviewing basic physicochemical aspects and role of hydrophobic interactions. Cellulose 19:581-587

Miao J, Sun H, Yu Y, Song X, Zhang L (2014) Quaternary ammonium acetate: an efficient ionic liquid for the dissolution and regeneration of cellulose. RSC Adv 4:36,721-36,724

Olsson C, Idström A, Nordstierna L, Westman G (2014) Influence of water on swelling and dissolution of cellulose in 1-ethyl-3-methylimidazolium acetate. Carbohydr Polym 99:438-446

Payal RS, Bejagam KK, Mondal A, Balasubramanian S (2015) Dissolution of cellulose in room temperature ionic liquids: anion dependence. J Phys Chem B 119:1654-1659

Pedersen JS, Schurtenberger P (1996) Scattering functions of semiflexible polymers with and without excluded volume effects. Macromolecules 29:7602-7612

Sellin M, Ondruschka B, Stark A (2010) hydrogen bond acceptor properties of ionic liquids and their effect on cellulose solubility. In: Liebert TF, Heinze TJ, Edgar KJ (eds) Cellulose solvents: for analysis, shaping and chemical modification. American Chemical Society, Washington, DC, pp 121-135

Sixta H, Michud A, Hauru L, Asaadi S, Ma Y, King AW, Kilpeläinen I, Hummel M (2015) Ioncell-F: a high-strength regenerated cellulose fibre. Nord Pulp Pap Res J 30:43-57

Stejskal EO, Tanner JE (1965) Spin diffusion measurements: spin echoes in the presence of a timedependent field gradient. J Chem Phys 42:288

Sun H, Miao J, Yu Y, Zhang L (2015) Dissolution of cellulose with a novel solvent and formation of regenerated cellulose fiber. Appl Phys A 119:539-546

Swatloski RP, Spear SK, Holbrey JD, Rogers RD (2002) Dissolution of cellose with ionic liquids. J Am Chem Soc 124:4974-4975

Tessier MB, DeMarco ML, Yongye AB, Woods RJ (2008) Extension of the glycam06 biomolecular force field to lipids, lipid bilayers and glycolipids. Mol Simul 34:349-363

Wang H, Gurau G, Rogers RD (2012) Ionic liquid processing of cellulose. Chem Soc Rev 41:1519-1537

Weingärtner $H$ (2008) Understanding ionic liquids at the molecular level: facts, problems, and controversies. Angew Chem Int Ed 47:654-670

Wilson PJ, Bradley TJ, Tozer DJ (2001) Hybrid exchangecorrelation functional determined from thermochemical data and ab initio potentials. J Chem Phys 115:9233

Zhang C, Kang H, Li P, Liu Z, Zhang Y, Liu R, Xiang J, Huang Y (2016) Dual effects of dimethylsulfoxide on cellulose solvating ability of 1-allyl-3-methylimidazolium chloride. Cellulose 23:1165-1175

Zhang J, Wu J, Yu J, Zhang X, He J, Zhang J (2017) Application of ionic liquids for dissolving cellulose and fabricating cellulose-based materials: state of the art and future trends. Mater Chem Front. doi:10.1039/c6qm00348f

Zhao Y, Liu X, Wang J, Zhang S (2013) Effects of anionic structure on the dissolution of cellulose in ionic liquids revealed by molecular simulation. Carbohydr Polym 94:723-730 